\title{
Gamificação e Autocuidado no Tratamento do Diabetes Tipo 2
}

\author{
Matheus F. C. Castro Rezende ${ }^{1}$, Luma W. Oliveira ${ }^{1}$, Sergio T. Carvalho ${ }^{1}$ \\ ${ }^{1}$ Instituto de Informática (INF) - Universidade Federal de Goiás (UFG) \\ mfilipeddiscente.ufg.br, \{luma_oliveira, sergiocarvalho\}@ufg.br
}

\begin{abstract}
The treatment of type 2 diabetes is often related to the patient's lifestyle. One of the great difficulties, however, is to keep the patient engaged for long-term adherence during their treatment. The games' ability to engage and persuade make it possible to create solutions that encourage self-care in health. This work presents a mobile application that uses gamification principles to engage the patient in the treatment of type 2 diabetes.
\end{abstract}

Resumo. $O$ tratamento do diabetes tipo 2 está, muitas vezes, relacionado ao estilo de vida do paciente. Uma das grandes dificuldades, no entanto, é a de manter o paciente engajado para uma adesão de longo prazo durante o seu tratamento. A capacidade de engajamento e de persuasão dos jogos torna possível criar soluções que estimulam o autocuidado em saúde. Esse trabalho apresenta uma aplicação móvel que utiliza princípios de gamificação para engajar o paciente no tratamento do diabetes tipo 2.

\section{Introdução}

O diabetes tipo 2 é uma doença crônica que evolui muitas vezes silenciosamente, sendo, portanto, de extrema importância o constante e contínuo monitoramento e cuidado do paciente, inclusive para que ele siga o tratamento de forma consistente para alcançar os resultados esperados [1]. O autocuidado vem como uma complementação ao cuidado, em que o paciente (com capacidade física e mental) cuida de sua própria saúde, o que potencializa a adesão ao tratamento desse paciente [4, 17].

Dessa forma, o uso de aplicações em saúde que utilizam tecnologias móveis voltadas para o autocuidado possui o objetivo de informar, notificar e monitorar o usuário para que ele cumpra as práticas de cuidado da sua saúde [14, 15]. Uma parcela dessas aplicações [11, 16] utiliza a gamificação como uma forma de engajar o usuário a utilizar a aplicação, e consequentemente incrementar a adesão ao tratamento. $\mathrm{O}$ termo gamificação designa "o processo de tornar as atividades mais semelhantes a jogos" [19] e utilizando elementos de jogos, para que o usuário se mantenha motivado em realizar atividades, sejam elas desgastantes, enjoativas, repetitivas ou mesmo dolorosas. A utilização da gamificação se torna propícia para o controle de doenças crônicas [10, 3], devido às características dessas doenças, ou seja, de duração longa ou incerta e cujo tratamento envolve mudanças de estilo de vida, em um processo de cuidado contínuo.

Este trabalho tem como objetivo investigar o uso da gamificação, considerando o perfil do usuário [7], por meio da implementação de uma aplicação móvel (mobile health - mHealth) que potencialize o engajamento do usuário na realização de atividades de autocuidado no tratamento do diabetes tipo 2. A base para o desenvolvimento desse trabalho é o Framework L [12, 6], um método de como gamificar aplicações para este fim. 
Além dessa seção introdutória, este artigo está estruturado em outras 3 seções: a Seção 2 descreve os conceitos envolvendo saúde e gamificação; a Seção 3 apresenta as funcionalidades e ferramentas da aplicação desenvolvida; e, por fim, a Seção 4 apresenta a conclusão e trabalhos futuros.

\section{Saúde e Gamificação}

\subsection{Diabetes e Autocuidado}

O diabetes tipo 2 se refere a um defeito na secreção da insulina associada a uma resistência a sua ação. Assim que a doença é diagnosticada, ela pode progredir por muitos anos antes que seja necessário o uso da insulina para controle [1, 18]. Dessa forma, é de extrema importância controlar a doença, e os resultados desse controle são obtidos através da soma de fatores e condições que propiciam o acompanhamento dos pacientes diabéticos, para os quais espera-se, além do controle da glicemia, o desenvolvimento do autocuidado.

Os principais objetivos das ações de saúde neste contexto são controlar a glicemia e, como resultado a longo prazo, reduzir a morbimortalidade causada por essa patologia. O controle metabólico mantém o paciente assintomático e previne as complicações agudas e crônicas da doença, resultando na melhoria da qualidade de vida [2]. O diabetes tipo 2 exige tratamento não farmacológico, complementado, em muitos casos, com antidiabético oral e, eventualmente, insulina basal, conforme a evolução da doença [1]. O tratamento tem os processos iniciais destacados por serem cruciais para evitar o desenvolvimento da doença.

Assim que a doença é diagnosticada, dá-se início às orientações para a mudança do estilo de vida (MEV) [1]. No decorrer de três meses do diagnóstico verifica-se as metas glicêmicas do paciente e se ele as atingiu; caso não tenha atingido, é introduzido um medicamento antidiabético oral. Após três meses, realiza-se outra avaliação das metas. Caso elas ainda não sejam atingidas, é introduzido um segundo medicamento antidiabético oral e se continuar sem resultados, o paciente é indicado para insulinoterapia. No caso do paciente alcançar as metas em qualquer uma das etapas, deve-se manter o acompanhamento de hemoglobina glicada de seis em seis meses.

O desenvolvimento de aplicações com o propósito de melhorar o engajamento do paciente a cuidar de suas taxas de glicemia vem sendo objeto de estudo em trabalhos da área [5]. Diante disso, é muito importante que o paciente monitore e acompanhe o nível de glicose, se os medicamentos estão sendo devidamente administrados, se a insulina está sendo aplicada ou não, e se a pessoa está praticando atividade física, controlando o peso e a alimentação.

\subsection{Gamificação}

Um jogo é caracterizado por recursos lúdicos que são capazes de manter um jogador envolvido. Huizinga [8] descreve esse envolvimento como um círculo mágico onde o jogador se ausenta do mundo "real": o caráter especial e excepcional de um jogo é ilustrado pelo ar de mistério em que frequentemente se envolve, fazendo com que as leis e costumes da vida cotidiana percam a sua validade. Nesse ambiente, a gamificação tem características que a fazem diferente de jogo. Werbach [19] define gamificação como "o processo de tornar as atividades mais semelhantes a jogos", para trazer o elemento lúdico como fundação para o uso dos elementos de jogos. Este trabalho considera a gamificação 
como uma estratégia de design que faz uso dos elementos de jogos para criar uma experiência lúdica, para engajar e mudar o comportamento do usuário.

A Gamificação Adaptativa, onde os elementos de jogo variam de acordo com o perfil do usuário, tem como principal objetivo apresentar ao usuário os elementos de jogos que reforçam suas motivações, de maneira personalizada [7]. Esse tipo de gamificação se diferencia do uso convencional dos elementos de jogos, já que cada usuário da mesma aplicação pode interagir com elementos diferentes. A Gamificação Adaptativa tem como motivação tornar a aplicação mHealth capaz de perceber que o usuário muda seus padrões de interação e que isso pode ser um sinal da mudança de interesse ou motivação [6, 7].

A classificação para os tipos de jogadores de Marczewski [9] descreve os usuários de aplicações gamificadas a partir de suas motivações. Além de delimitar os perfis de usuários, ela também descreve quais elementos de jogos atraem mais cada perfil de jogador, dessa forma, ele relaciona perfil de jogador e elementos de jogos. Os elementos de jogos escolhidos neste trabalho foram: tarefas, pontuação, níveis, medalhas, ranking e fórum de discussão. São seis os perfis de usuários da classificação de Marczewski [9]: Achiever, Disruptor, Free-Spirit, Philantropist, Player, e Socialiser. Esse estudo de Marczewski é um ponto importante na gamificação adaptativa por ser uma justificativa da escolha dos elementos de jogos baseada nos perfis de jogadores [6].

Tabela 1. Funcionalidades implementadas.

\begin{tabular}{|l|l|}
\hline Função & Recursos \\
\hline Controle glicêmico & $\begin{array}{l}\text { Alarme sonoro } \\
\text { Registro de medidas } \\
\text { Premiação pelo alcance de metas de glicemia }\end{array}$ \\
\hline Insulinoterapia & $\begin{array}{l}\text { Alarme sonoro } \\
\text { Registro de aplicação }\end{array}$ \\
\hline Atividade física & $\begin{array}{l}\text { Registro de prática } \\
\text { Alarme sonoro }\end{array}$ \\
\hline Abordagem dos sentimentos & Uso de emoticons \\
\hline Relações sociais & Gráficos com registros semanais das taxas de glicemia \\
\hline
\end{tabular}

\section{Desenvolvimento da aplicação}

A aplicação possui a funcionalidade de registro, que serve para o paciente fazer anotações relacionadas à glicemia, insulina, medicamentos orais, atividades físicas e peso (relacionado com o Índice de Massa Corporal, IMC). Destaque para a avaliação qualitativa do humor do paciente que é registrado para um histórico de humor. Há também a funcionalidade de alarmes, que tem como objetivo notificar (lembrar) o usuário das suas atividades, com o objetivo de mantê-lo engajado. As funcionalidades implementadas na aplicação estão descritas na Tabela 1 e ilustradas na Figura 1.

Para o desenvolvimento da gamificação baseada em tipos de jogadores, foi implementado um sistema de recompensas por pontuação juntamente com o conceito de níveis. Dessa forma, o paciente ganha pontos ao realizar seus registros. O acúmulo de pontos faz ele progredir de nível. Além disso, a aplicação conta com um sistema de medalhas, que são obtidas ao realizar tarefas relacionadas aos registros de saúde, tornando esse processo mais lúdico e interativo. Por fim, foi implementado um ranking mostrando os usuários mais ativos na aplicação. Algumas dessas funcionalidades são apresentadas na Figura 2. 


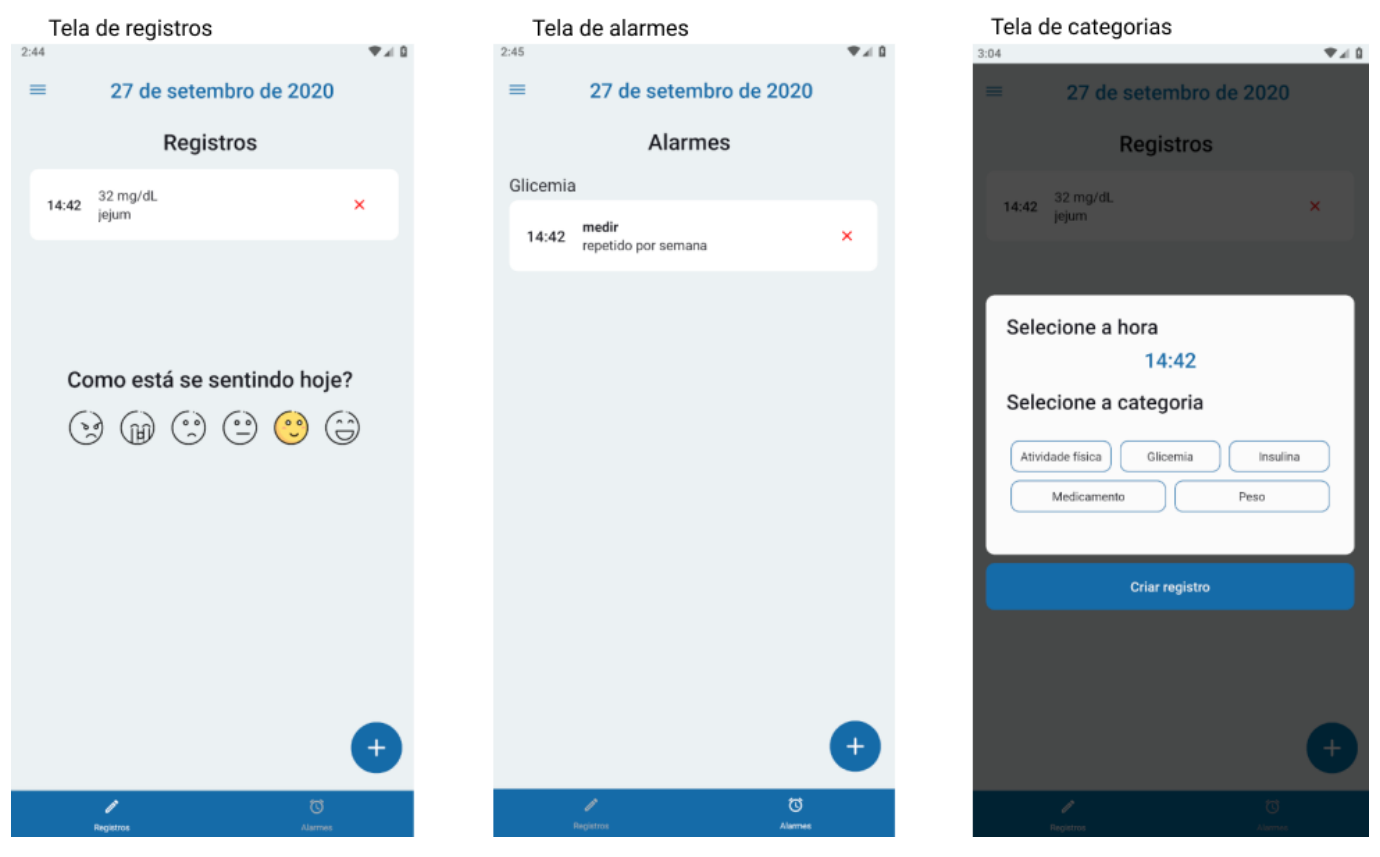

Figura 1. Funcionalidades de registros, alarmes e categorias.

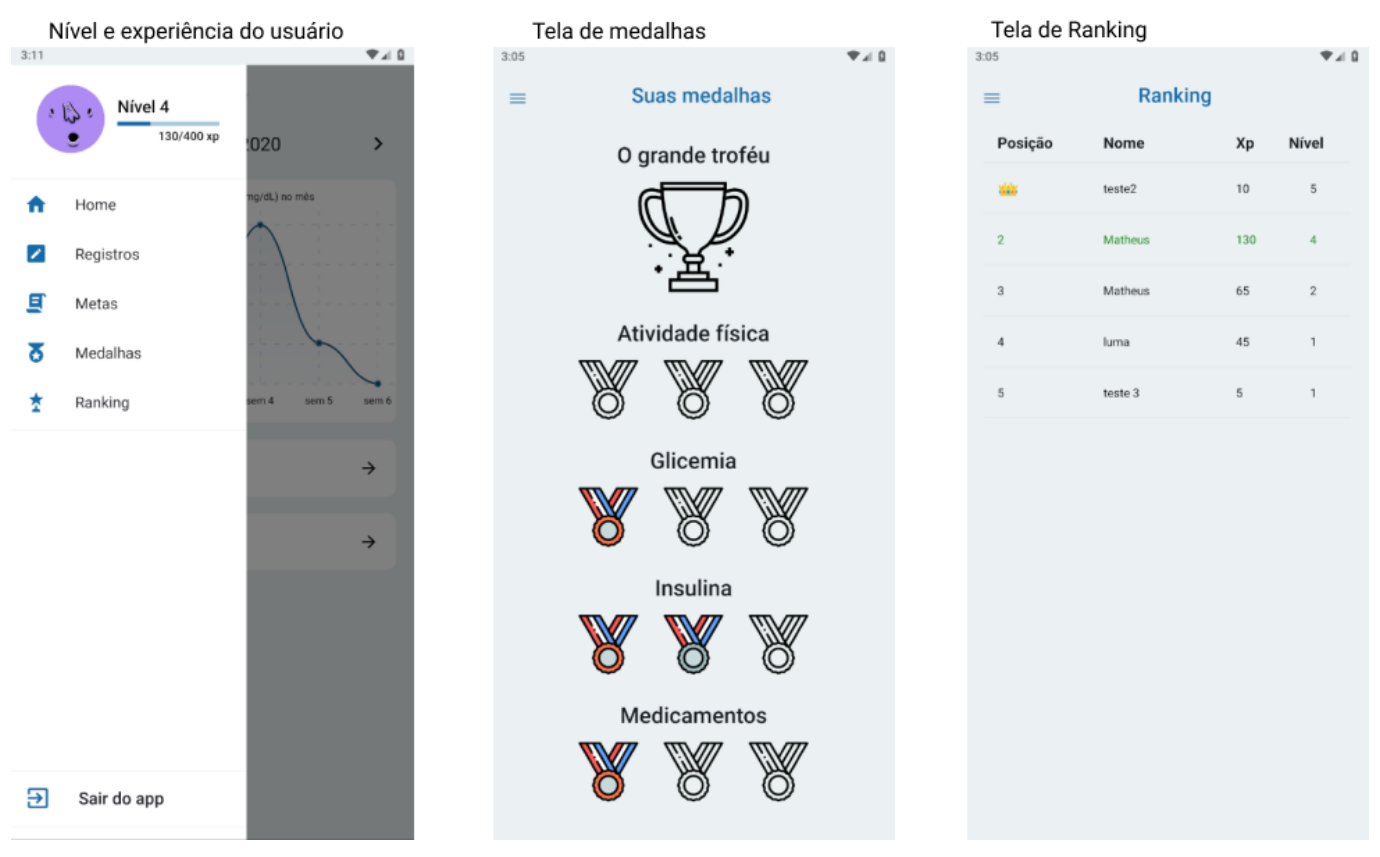

Figura 2. Funcionalidades de nível do usuário, medalhas e ranking.

Esses elementos reforçam as motivações do tipo de jogador Player, que são pessoas motivadas pela recompensa, do tipo Achiever, que são pessoas que se sentem motivadas pelo domínio e autoaperfeiçoamento, do tipo Socialiser, que são pessoas motivadas pelos relacionamentos sociais e do tipo Free-Spirit que são motivados pela exploração e independência no uso da aplicação [9]. Dessa forma, diferentes perfis de usuários se sentem engajados a cuidar de sua saúde, potencializando sua adesão no tratamento da doença 
crônica.

O usuário possui acesso a um gráfico detalhado de seus registros de glicose no mês selecionado, separado por semana, para que ele possa monitorar seu progresso com o controle de glicose. Ele também tem acesso a uma tabela detalhada sobre seu IMC. Essas funcionalidades são mostradas na Figura 3.

O código-fonte da aplicação está disponível em https://bityli.com/HbbjY.
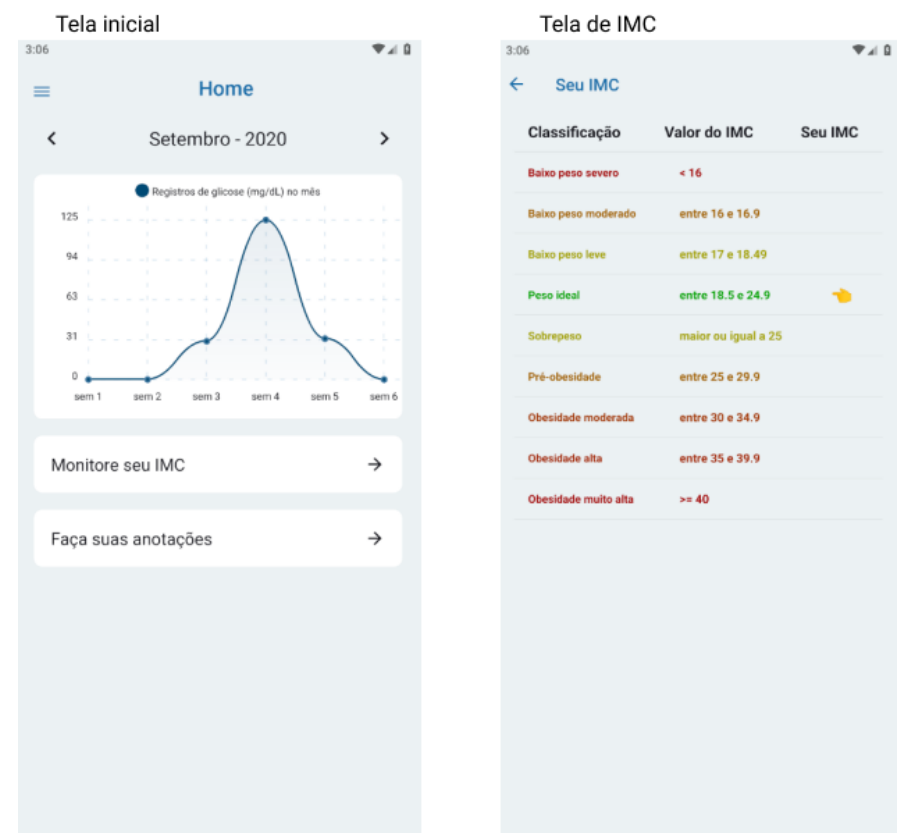

Figura 3. Página principal da aplicação e tela de IMC

\section{Conclusão}

Este trabalho apresentou uma aplicação móvel desenvolvida que utiliza técnicas de gamificação por meio de elementos de jogos para engajar o paciente a praticar o autocuidado para o tratamento do diabetes tipo 2. É importante mencionar que o grupo de pesquisa onde os autores atuam possuem um framework com diretrizes para o desenvolvimento de aplicações móveis gamificadas em saúde [6, 12], utilizado na concepção da aplicação. Em fase de testes e validação, a aplicação integra um projeto mais amplo conduzido no grupo de pesquisa em computação aplicada à saúde no INF/UFG. Nesse processo, o desenvolvimento de um serviço backend que possa disponibilizar e tratar os dados da aplicação está em construção, uma vez que para a efetiva implementação foi utilizada uma base de dados simulada. Os próximos passos incluem a avaliação da usabilidade, testes com pacientes e o uso de inteligência artificial para se conseguir uma experiência de usuário mais associada ao que se espera da gamificação adaptativa [13, 6, 12].

\section{Referências}

[1] Min da saúde, atenção básica. estratégias para o cuidado da pessoa com doença crônica: diabetes mellitus, (2013). Cadernos de Atenção Básica, n. 36, 2013.

[2] Standards of medical care in diabetes-2013, Diabetes Care, 36 (2013), pp. S11-S66. 
[3] A. Almarshedi, G. B. Wills, And A. Ranchhod, The wheel of sukr: A framework for gamifying diabetes self-management in saudi arabia, PCS, 63 (2015), pp. $475-480$.

[4] C. CAmargo-Borges And M. JAPUR, Sobre a não adesão ao tratamento: ampliando sentidos do autocuidado, Texto \& Contexto - Enfermagem, 17 (2008), pp. 64 - 71.

[5] F. F. Chaves, T. L. A. D. Carvalho, E. C. Paraíso, A. S. Pagano, I. A. Reis, and H. C. TORRES, Aplicativos para adolescentes com diabetes mellitus tipo 1: revisão integrativa da literatura, Acta Paulista de Enfermagem, 30 (2017), pp. 565-572.

[6] L. W. DE Oliveira, Framework l para desenvolvedores de mhealth no contexto de self-care e gamificação, Master's thesis, Universidade Federal de Goiás, 2018.

[7] L. W. De Oliveira And S. T. De CARVAlho, Gamificação adaptativa: uma abordagem para incentivar o engajamento, in Simp Bras Jogos e Entretenimento Digital, 2020, pp. 155-164.

[8] J. Huizinga, Homo ludens, (2000). volume 3. Perspectiva, 2001.

[9] A. MARCZEwski, Even ninja monkeys like to play: Gamification, Game Thinking \& Motivational Design, (2015).

[10] A. S. Miller, J. A. Cafazzo, And E. Seto, A game plan: Gamification design principles in mhealth applications for chronic disease management, Health Inf Journal, 22 (2016), pp. 184-193.

[11] F. Nunes, N. Verdezoto, G. Fitzpatrick, M. Kyng, E. Gr"onvall, and C. Storni, Self-care technologies in hci: Trends, tensions, and opportunities, 22 (2015).

[12] L. OliveIRA AND S. CARvalho, Framework l para desenvolvedores de mhealth no contexto de self-care e gamificação, in Simp Bras de Computação Aplicada à Saúde, 2019, pp. 61-66.

[13] L. Oliveira, S. De Lima Vieira dos Santos, and S. T. Carvalho, Salus ciber ludens: jogo aplicado para o autocuidado em diabetes em uma experiência conectante, in SBGAMES 2020 - Trilha de Saúde, nov 2020.

[14] H. Ribeiro, D. Battisti, E. Germano, and S. Carvalho, Notificações de monitoramento remoto de pacientes usando redes sociais, 112016.

[15] H. Ribeiro, E. Germano, S. Carvalho, and E. Albuquerque, Integrating social networks and remote patient monitoring systems to disseminate notifications, vol. 245, 08 2017.

[16] K. Rose, M. Koenig, And F. Wiesbauer, Evaluating success for behavioral change in diabetes via mhealth and gamification: Mysugr's keys to retention and patient engagement, Diabetes Technology \& Therapeutics, 15 (2013).

[17] I. D. J. Silva AND E. A. Oliveira, Cuidado, autocuidado e cuidado de si: uma uma compreensão paradigmática para o cuidado de enfermagem, Revista da Escola de Enfermagem da USP, 43 (2009), pp. 697 - 703.

[18] G. S. TRES, Efeito da diacereína nos parâmetros inflamatórios e controle metabólico em pacientes diabéticos tipo 2 em uso de anti-hiperglicemiantes, (2015).

[19] K. WERBACH, (re)defining gamification: A process approach, vol. 8462, 05 2014, pp. 266-272. 